\title{
REVIEW
}

\section{Year in review 2011: Critical Care - Out-of-hospital cardiac arrest and trauma}

\author{
Scott A Goldberg*, Jeffery C Metzger and Paul E Pepe
}

\begin{abstract}
In 2011, numerous studies were published in Critical Care focusing on out-of-hospital cardiac arrest, cardiopulmonary resuscitation, trauma, and some related airway, respiratory, and response time factors. In this review, we summarize several of these studies, including those that brought forth advances in therapies for the post-resuscitative period. These advances involved hypothesis-generating concepts in therapeutic hypothermia as well as the impact of early percutaneous coronary artery interventions and the potential utility of extracorporeal life support after cardiac arrest. There were also articles pertaining to the importance of timing in prehospital airway management, the outcome impact of hyperoxia, and the timing of end-tidal carbon dioxide measurements to predict futility in cardiac arrest resuscitation. In other articles, additional perspectives were provided on the classic correlations between emergency medical service response intervals and outcomes.
\end{abstract}

\section{Introduction}

During the year 2011, many innovative and thoughtprovoking resuscitation studies were published in Critical Care. A distinct portion of these articles focused on outof-hospital cardiac arrest (OHCA) and cardiopulmonary resuscitation $(\mathrm{CPR})$, ranging from emergency medical service (EMS) response intervals and airway management in both trauma and cardiac arrest cases to the early use of percutaneous coronary artery intervention (PCI) and extracorporeal life support (ECLS), and several of the articles pertained to respiratory factors in CPR. Many of the studies discussed the timing of interventions, including the effect of the time to reach target temperature in therapeutic hypothermia.

*Correspondence: scott.goldberg@utsouthwestern.edu

Emergency Medicine Administration, University of Texas Southwestern Medical Center at Dallas, 5323 Harry Hines Boulevard, MC 8579, Dallas, TX 75390-8579, USA

\section{Time intervals to reach target core temperature and outcome following induced hypothermia after resuscitation from cardiac arrest}

Despite recent improvements in enhancing successful resuscitation from OHCA, overall long-term outcomes remain poor in most venues [1,2]. One well-touted intervention that has been shown to have benefit in recent years is the use of post-resuscitative mild therapeutic hypothermia (MTH), showing both improved neurologic outcomes and long-term survival [3-6]. Consensus guidelines for post-arrest management now advocate its use in survivors of OHCA, particularly those who present with ventricular dysrhythmias [7]. Nevertheless, the ideal timeline for inducing the core temperature goal in $\mathrm{MTH}$ remains elusive $[6,8]$.

This past year in Critical Care, Haugk and colleagues [9] sought to address this question in a single-center retrospective cohort study. The investigators evaluated 2,536 OHCA patients, of whom $23 \%$ (588) underwent MTH with a target core temperature of less than $34^{\circ} \mathrm{C}$. The authors divided subjects into three groups: those arriving at the goal temperature (a) in less than 120 minutes, (b) in 120 to 220 minutes, and (c) more than 220 minutes. They defined the achievement of a good neurologic outcome as having a Pittsburgh Cerebral Performance Category (CPC) score of 1 or 2 (normal or only mild impairment) at 6-month follow-up [9].

The authors found that patients reaching the goal temperature faster had significantly worse 6-month neurologic outcomes (odds ratio (OR) 1.86, 95\% confidence interval (CI) 1.03 to 3.38) but that the time taken to achieve the goal temperature had no association with 6-month all-cause survival [9]. At first glance, these findings call into question guidelines advocating rapid cooling $[8,10]$.

However, while it is indeed possible that faster cooling was directly causal in the poorer neurologic outcomes, it is more likely that the adverse outcomes were simply a reflection of more pronounced cerebral and systemic insults in these patients. In this line of thinking, those severe insults resulted in dysfunction of cerebral and systemic thermoregulation and thus a more rapid cooling. 
This study was clearly a provocative and hypothesisgenerating addition to the critical care literature. While one might argue that their findings prompt the need for prospective controlled evaluations of alternative timelines for inducing hypothermia, the study also reinforced the universal need for scientists and clinicians to be cautious with the face-value interpretations of results.

\section{Impact of adding percutaneous coronary artery intervention as part of a routine post-resuscitation care bundle following cardiac arrest}

Cardiac arrest may result from numerous precipitating factors involving both cardiac and non-cardiac etiologies. However, the most common cause of OHCA is ongoing myocardial ischemia leading to a lethal cardiac dysrhythmia such as ventricular fibrillation (VF) [11]. Even when resuscitative efforts lead to a successful return of spontaneous circulation (ROSC), OHCA patients still have a poor prognosis in many cases.

One root cause of the cardiac arrest and subsequent post-resuscitation morbidity and mortality is ongoing myocardial ischemia, most often a result of reversible coronary artery occlusion. While previous studies have shown no benefit to systemic thrombolysis, there does appear to be some advantage to a more direct coronary intervention, regardless of initial electrocardiogram (ECG) findings [12,13]. Furthermore, studies have indicated the outcome advantages of PCI after OHCA either as an individual intervention [14] or performed in combination with MTH [14-16].

In an attempt to further confirm the bundled effects of PCI and MTH on outcomes after OHCA, Gräsner and colleagues [17] retrospectively analyzed the German Resuscitation Registry. The clinical course and outcomes of 584 patients who had achieved ROSC and hospital admission - out of 2,973 cases of OHCA - were analyzed. Primary outcomes examined included (a) 24-hour survival and (b) a CPC score of 1 or 2 at the time of hospital discharge. Of the 584 admitted patients, 81 (14\%) received PCI alone, $73(12.5 \%)$ received both PCI and $\mathrm{MTH}$, and 106 (18\%) received MTH alone. Of the 154 (26\%) patients receiving PCI, 95\% were considered to have had an arrest of presumed cardiac etiology, $66 \%$ of which were associated with initial ECG rhythms of VF or ventricular tachycardia (VT).

The results demonstrated that patients receiving only MTH had significant increases in 24-hour survival (OR 7.02, 95\% CI 3.7 to 13.3) and neurologic function (OR $2.21,95 \%$ CI 1.23 to 3.96 ) as compared with the other groups. Likewise, patients receiving only PCI showed significant improvement in 24-hour mortality (OR 5.6, 95\% CI 2.63 to 9.71) and neurologic outcomes (OR 11.31, 95\% CI 6.25 to 20.47). Among patients receiving both interventions, significant improvements were again observed in 24-hour mortality (OR 7.6, 95\% CI 4.32 to 13.37) and neurologic outcomes (OR 1.83, 95\% CI 1.23 to 2.74). However, after correction for confounding variables, the addition of MTH to PCI only improved 24-hour survival but not neurologic outcomes [17].

Needless to say, the retrospective nature of this article leads to questions about selection bias, particularly a 'survival bias' (for example, patients surviving long enough to receive PCI obviously had a better chance of receiving the intervention and thus the correlation with survival). Nevertheless, despite those potential limitations, the data still seem to reconfirm the likely advantage of PCI, including the possible synergy of the bundled approach of combining MTH with PCI following OHCA [18]. Moreover, this article helps to support the potential benefit of PCI for all patients with OHCA of presumed cardiac etiology regardless of initial ECG findings [13].

\section{Oxygenation levels and end-tidal carbon dioxide concentrations during cardiopulmonary resuscitation efforts}

Published literature suggests that changes in oxygen saturation during resuscitation from cardiac arrest may affect outcome $[19,20]$. Whereas animal studies have shown an association between hyperoxia and increased mortality, results of human studies are less clear [21]. A 2010 study by the Emergency Medicine Shock Research Network (EMShockNet) research group showed an independent and stepwise relationship between hyperoxia and in-hospital mortality after resuscitation from cardiac arrest [20]. In an attempt to validate that work, Bellomo and colleagues [22] identified all of the blood gas analyses obtained during the first 24 hours of intensive care unit (ICU) admission for all survivors of cardiac arrest over a decade (2000 to 2009) by using a large Australian-New Zealand database. The authors published their findings in Critical Care in 2011 [22].

In this study, relative 'hypoxia' was defined as an arterial partial pressure of oxygen $\left(\mathrm{PaO}_{2}\right)$ of less than $60 \mathrm{~mm} \mathrm{Hg}$ or $\mathrm{PaO}_{2} /$ fraction of inspired oxygen $\left(\mathrm{PaO}_{2} /\right.$ $\mathrm{FiO}_{2}$ ) ratio of less than $300 \mathrm{~mm} \mathrm{Hg}$ whereas 'hyperoxia' was defined as a $\mathrm{PaO}_{2}$ of more than $300 \mathrm{~mm} \mathrm{Hg}$. All other patients were classified as 'normoxic'. Primary outcomes evaluated included in-hospital mortality and time to death in hours. A secondary outcome was successful discharge to home. Among the 12,806 patients who met inclusion criteria, $5.4 \%$ were subsequently excluded because of missing data or ICU readmission and 58\% $(6,968)$ died in the hospital.

In terms of outcomes, there was a significant survival benefit for those with normoxia versus hypoxia or hyperoxia $(P<0.0001)$. Of these survivors, $65 \%$ were ultimately discharged to home. However, once injury severity was added to a regression model, the effect on 
in-hospital mortality was markedly decreased (OR 1.2, 95\% CI 1.1 to 1.4 for hypoxia and OR 1.2, 95\% CI 1.0 to 1.5 for hyperoxia), and hyperoxia no longer showed a statistically significant association with discharge to home. Furthermore, the results demonstrated that an increased overall survival rate (65\%), as compared with that of the previous EMShockNet study (44\%), was possibly explained by a very high rate of MTH (median temperature of $34.9^{\circ} \mathrm{C}$ ) in this cohort.

Whereas previous research has suggested an association between hyperoxia and poorer outcomes, this retrospective analysis by Bellomo and colleagues [22] demonstrated much weaker associations [20]. Recognizing the more concerning potential for hypoxia by restricting inspired $\mathrm{FiO}_{2}$, the investigators recommended that clinicians take caution in ordering deliberate decreases in $\mathrm{FiO}_{2}$ to avoid hyperoxia and that related protocols be revisited.

Another respiratory parameter that has been widely used in the management of CPR has been the quantitative monitoring of end-tidal carbon dioxide $\left(\mathrm{ETCO}_{2}\right)$. $\mathrm{ETCO}_{2}$ has gained extensive support for monitoring advanced airway patency as well as the quality of CPR. It also has been used to predict the likelihood of successful resuscitation and thus used to help invoke termination of resuscitative efforts [10,23-26]. However, Grmec and colleagues [27] also demonstrated a significant difference in initial $\mathrm{ETCO}_{2}$ between asphyxial cardiac arrest and arrest secondary to VF/VT. In this case, the initial $\mathrm{ETCO}_{2}$ was not a valid predictor of ROSC [27].

Accordingly, in a 2011 Critical Care publication, Lah and colleagues [28] reported a prospective evaluation of 325 OHCA patients between 2006 and 2009 at a single center in Slovenia. After the exclusion of 211 patients for various reasons, the remaining patients were divided into two groups: (a) 51 patients presenting with asphyxial arrest and an initial cardiac rhythm of asystole or pulseless electrical activity (PEA) and (b) 63 patients with cardiac arrest of presumed cardiac etiology and an initial rhythm of VF or pulseless VT. ETCO ${ }_{2}$ measurements were made immediately following endotracheal intubation (ETI) and every minute thereafter. Interestingly, initial $\mathrm{ETCO}_{2}$ values were significantly higher in the asphyxial group $(6.74 \pm 4.22 \mathrm{kPa}$ versus $4.51 \pm 2.47 \mathrm{kPa})$, but this difference was no longer significant after 3 minutes of CPR. In addition, patients achieving successful ROSC had an initial $\mathrm{ETCO}_{2}$ of more than $1.33 \mathrm{kPa}$, a finding consistent with established literature describing the futility of extended CPR in patients with lower initial levels [24-26].

One of the key findings of this study, however, was that the $\mathrm{ETCO}_{2}$ level was the most predictive of ROSC after the fifth minute of CPR (asphyxial group: $6.09 \pm 2.63 \mathrm{kPa}$ versus $4.47 \pm 3.35 \mathrm{kPa}, P=0.006 ; \mathrm{VF} / \mathrm{VT}$ group:
$5.63 \pm 2.01 \mathrm{kPa}$ versus $4.26 \pm 1.86 \mathrm{kPa}, P=0.015)$. Thus, this important work indicates that the association between $\mathrm{ETCO}_{2}$ and ROSC should be established after 5 minutes of CPR performed in accordance with published guidelines [29].

\section{Extracorporeal life support for out-of-hospital cardiac arrest}

In recent years, ECLS has been proposed as an effective therapy not only for in-hospital cardiac arrest but also for OHCA [30-36]. However, outcomes with this novel therapy have been mixed. Le Guen and colleagues [37] added to this growing body of literature in a 2011 Critical Care article describing their ECLS experience with 51 OHCA patients whose arrest was witnessed but ROSC was not achieved after 30 minutes of resuscitative efforts and the institution's mobile cardiothoracic surgery team was available. In cases of refractory cardiac arrest, an automated compression device was used. ECLS was established by using femoral-femoral cannulation, and the mean time to ECLS was 120 minutes from initiation of CPR. All patients also received 24 hours of MTH.

Of the 51 patients meeting inclusion criteria, 9 (18\%) failed successful cannulation and only $17(42 \%)$ were alive at 24 hours. Two ( $4 \%$ of the total 51 candidates) survived to day 28 (95\% CI $1 \%$ to $13 \%$ ). Both surviving patients had a no-flow time of less than 5 minutes but a low-flow time of more than 100 minutes. One was successfully weaned at day 5, but the other required 36 days. Both patients had a Glasgow Outcomes Scale score of 4 or 5 (relatively favorable outcomes) at 28 days and 6 months [37].

Although this study by Le Guen and colleagues [37] showed lower survival rates than those of previous studies, both survivors had favorable neurologic outcomes and $88 \%$ of non-survivors expired within 48 hours, contradicting the fear that ECLS may unnecessarily prolong life in futile cases. Also, the long (2-hour) mean initiation time may have contributed to these poorer outcomes. Therefore, this study supports the potential benefits of ECLS for OHCA and suggests important steps for improvements in ECLS protocols such as earlier implementation [37].

\section{Additional perspectives on emergency medical service response intervals and outcomes following cardiopulmonary arrest}

Shorter elapsed time intervals from collapse to initiation of CPR have been established as important contributors to favorable outcomes following OHCA [38-40]. However, most of these studies have focused on OHCA of presumed cardiac etiology. Koike and colleagues [41] used a large nationwide database to examine undifferentiated OHCA and described their findings in a 2011 
Critical Care article. By means of logistic regression techniques, 109,350 cases in which the collapse was witnessed by bystanders were analyzed. Only half of the cases were of presumed cardiac etiology. Bystanderperformed CPR was initiated in $45 \%$ of cases, and an automated external defibrillator (AED) was used in $0.8 \%$. The mean collapse to EMS CPR interval (CECI) was 14.5 minutes.

The authors demonstrated a strong correlation between CECI and 1-month survival $\left(\mathrm{R}^{2}=0.98\right)$ and 1-month CPC scores of 1 or $2\left(R^{2}=0.95\right)$. Consistent with existing literature, 1-month survival and good neurologic outcomes were associated with a cardiac etiology, bystander CPR, and public AED use [42-44]. In essence, this study confirmed existing information, but with a much larger and diverse data set, and strongly reinforces the critical importance of public access to AEDs as well as community training in bystander CPR [45].

Another perspective on response intervals was provided this past year in Critical Care by Neukamm and colleagues [46], who used the German Resuscitation Registry, a national OHCA database established by the German Society for Anesthesiology and Intensive Care to evaluate 'response time reliability' (RTR) on patient survival to hospital admission [46,47]. RTR was defined as the percentage of cases in which the first responding EMS crew arrived on the scene within 8 minutes of the call for help [46].

The group examined 2,330 cases over several years in seven selected EMS systems servicing a variety of population densities in Germany. All systems performed resuscitation based on 2005 guidelines [29]. Of the cases examined, $46.7 \%$ achieved ROSC in the field, $42.8 \%$ survived to hospital admission, and $15.4 \%$ survived to hospital discharge. As expected, there was a significant association between higher RTR and survival to hospital admission (24.4/100,000 population/year for RTR of more than $70 \%$ versus $15.6 / 100,000$ population/year for RTR of less than $70 \%$, OR $1.57,99 \%$ CI 1.274 to 1.935 ), although no significant differences were found in rates of ROSC. However, all seven EMS systems achieved higherthan-expected rates of ROSC based on resuscitation after cardiac arrest (RACA) scores $[47,48]$. This finding was possibly due to the high utilization of therapeutic hypothermia in these seven centers (46.2\% versus $13.7 \%$ across the entire region).

The frequency of bystander-initiated CPR was low overall and showed great variability across systems, ranging from $1.3 \%$ to $28.6 \%(P<0.001)$. The two largest cities had the fastest response times $(67.9 \%$ and $64.2 \%$ of resuscitations were initiated within 8 minutes), whereas two other systems achieved RTR in only $56 \%$ and $53 \%$ of cases $(P<0.001)$. The less reliable systems had the lowest frequencies of CPR performance as well. In essence, these findings indicated specific areas for improvement in terms not only of achieving RTR targets but of frequency of bystander CPR. In addition, it should be noted that 8 minutes is an arbitrary target, and further delineation of even shorter RTRs may be worthwhile.

\section{Evolving insights on prehospital airway management}

Airway management in OHCA has been the topic of much debate in recent years. ETI has long been considered the primary advanced airway maneuver, but their benefit over extraglottic airways (EGAs) in the OHCA setting has become uncertain for many medical decision makers [49]. In addition, the optimal time in which an advanced airway should be placed has not been defined. Therefore, in a 2011 Critical Care article, Kajino and colleagues [50] compared outcomes with EGA and ETI use by EMS personnel for the treatment of witnessed non-traumatic OHCA in Osaka, Japan, and also examined the timeline of placement of the airway and its relationship to outcomes.

Of 5,377 adult patients meeting inclusion criteria, there were no significant differences in 1-month survival chances between EGA (9.8\%) and ETI (10.7\%) and no significant difference in 1-month neurologic outcomes between the EGA (3.6\%) and ETI (3.6\%). However, there was a statistically significant difference in ROSC between EGA placement (10.1\%) and ETI (16.6\%). In context though, only advanced practitioners with additional training are allowed by protocol to give catecholamines and place endotracheal tubes. Therefore, the addition of catecholamines may explain this improved ROSC despite the similar long-term outcomes. Furthermore, the use of these advanced practitioners had an independent association with favorable neurologic outcomes (OR 1.86, 95\% CI 1.04 to 3.34 ).

Still, one of the most interesting findings of this study was the effect of time to establishment of an advanced airway and outcome. The investigators found a statistically significant decrease in favorable neurologic outcome with each passing minute (adjusted OR 0.91, $95 \%$ CI 0.88 to 0.95 ). So these data seem to indicate that, if an advanced airway is to be placed, early placement may very well be beneficial. However, others may caution that the early airway placement would be acceptable but only assuming that there are no significant interruptions to the performance of high-quality chest compressions [50,51].

In the realm of trauma care, while advanced airway management is typically part of the protocol in severe injury, the advantages of prehospital ETI for more moderate trauma are less clear [52,53]. In a 2011 Critical Care article, Hussman and colleagues [54] examined the German Association for Trauma Surgery database from 
2005 to 2008 to evaluate the impact of prehospital ETI on moderately injured patients. The authors performed a matched-pairs analysis of 600 patients from Austria or Germany who were undergoing prehospital ETI, were more than 16 years old, had a Glasgow Coma Scale score of 13 to 15 and maximum injury severity per body region (Abbreviated Injury Score) of not more than 3, and who received no packed red blood cells in the emergency department. Patients were matched to 600 non-intubated controls.

The authors found no significant difference in survival to hospital discharge between groups. However, mortality in both groups was quite low: 1\% (ETI) and 0.5\% (control). Both groups also had low rates of emergency operative intervention. However, the ETI patients had significantly higher levels of volume replacement; decreases in hemoglobin, prothrombin time, and platelet count; and increased levels of hypothermia. They also had significantly lower blood pressures and higher heart rates at hospital admission and received more prehospital invasive procedures, including chest tubes and CPR. Furthermore, the ETI group had a significantly higher rate of multi-organ failure and sepsis. Finally, intubated patients had significantly longer ICU and total hospital stays and a $26.1 \%$ higher hospital cost.

These findings may reflect simply selection bias and inadequate case controls. Also, the sympatholytic properties of sedatives and paralytics associated with intubation may explain the variation in vital signs [55]. In addition, ETI patients received significantly more volume replacement, which may have contributed to hypothermia, clotting factor dilution, and decreases in hemoglobin and platelet counts [56]. Furthermore, the detrimental effects of positive pressure ventilation in the face of relative hypovolemia could also affect blood pressure and pulse. Likewise, the incidence of CPR was higher in the intubated cohort, again reflecting a 'sicker' population.

At first glance, therefore, this study may give pause to using ETI in moderately injured patients, but one might argue that the results reflect that a sicker population had received ETI and that it may even have been beneficial considering the relative equality in outcomes. The jury is still out, but this article gives us more concepts to consider in this ongoing debate.

\section{Conclusions}

In articles published in Critical Care in 2011, out-ofhospital cardiac arrest and trauma care were central topics of research. Authors added to the growing evidence in support of PCI for survivors of OHCA, and time to goal temperature for MTH was found to be a complicated issue. Concerns over hyperoxia in cardiac arrest survivors were again raised, although the impact of this finding may not be as clear as previously thought. The ability of $\mathrm{ETCO}_{2}$ measurement to predict return of pulse was further defined in terms of timelines for making decisions to terminate resuscitative efforts. The articles also provide some evidence for ECLS as a potential intervention for OHCA. Authors further highlighted the importance of faster responses by prehospital care providers, bystander CPR, and early AED use in the management of OHCA and provided new perspectives such as response time reliability. Finally, it appears that the earliest placement of an advanced airway is important and that extraglottic airways may be a useful alternative to ETI in OHCA. However, in the moderately injured patient, providers may need to be more conservative in their decision to place an endotracheal tube. Nevertheless, depending upon the patient, ETI may still have important utility in trauma care.

\section{Abbreviations}

$A E D$, automated external defibrillator; $C E C l$, collapse to emergency medical services cardiopulmonary resuscitation interval; $\mathrm{Cl}$, confidence interval; CPC, Pittsburgh Cerebral Performance Category; CPR, cardiopulmonary resuscitation; ECG, electrocardiogram; ECLS, extracoporeal life support; EGA, extraglottic airway; EMS, emergency medical services; EMShockNet, Emergency Medicine Shock Research Network; ETCO ${ }_{2}$, end-tidal carbon dioxide; $\mathrm{ETI}$, endotracheal intubation; $\mathrm{FiO}_{2}$, fraction of inspired oxygen; ICU, intensive care unit; MTH, mild induced therapeutic hypothermia; OHCA, out of hospital cardiac arrest; $\mathrm{OR}$, odds ratio; $\mathrm{PaO}_{2}$, arterial partial pressure of oxygen;

$\mathrm{PCl}$, percutaneous coronary intervention; ROSC, return of spontaneous circulation; RTR, response time reliability; VF, ventricular fibrillation; VT, ventricular tachycardia.

\section{Competing interests}

The authors declare that they have no competing interests.

Published: 10 December 2012

\section{References}

1. Bigham BL, Koprowicz K, Rea T, Dorian P, Aufderheide TP, Davis DP, Powell J, Morrison LJ; ROC Investigators: Cardiac arrest survival did not increase in the Resuscitation Outcomes Consortium after implementation of the 2005 AHA CPR and ECC guidelines. Resuscitation 2011, 82:979-983.

2. Deasy C, Bray JE, Smith K, Wolfe R, Harriss LR, Bernard SA, Cameron P: Cardiac arrest outcomes before and after the 2005 resuscitation guidelines implementation: evidence of improvement? Resuscitation 2011 , 82:984-988.

3. Bernard SA, Gray TW, Buist MD, Jones BM, Silvester W, Gutteridge G, Smith K: Treatment of comatose survivors of out-of-hospital cardiac arrest with induced hypothermia. N Eng/ J Med 2002, 346:557-563.

4. Holzer M, Bernard SA, Hachimi-Idrissi S, Roine RO, Sterz F, Müllner M; Collaborative Group on Induced Hypothermia for Neuroprotection After Cardiac Arrest: Hypothermia for neuroprotection after cardiac arrest: systematic review and individual patient data meta-analysis. Crit Care Med 2005, 33:414-418.

5. Arrich J, Holzer M, Herkner H, Müllner M: Cochrane corner: hypothermia for neuroprotection in adults after cardiopulmonary resuscitation. Anesth Analg 2010, 110:1239-1240.

6. Testori C, Sterz F, Behringer W, Haugk M, Uray T, Zeiner A, Janata A, Arrich J, Holzer M, Losert H: Mild therapeutic hypothermia is associated with favourable outcome in patients after cardiac arrest with non-shockable rhythms. Resuscitation 2011, 82:1162-1167.

7. Peberdy MA, Callaway CW, Neumar RW, Geocadin RG, Zimmerman JL, Donnino M, Gabrielli A, Silvers SM, Zaritsky AL, Merchant R, Vanden Hoek TL, Kronick SL: Part 9: post-cardiac arrest care: 2010 American Heart Association Guidelines for Cardiopulmonary Resuscitation and Emergency Cardiovascular Care. Circulation 2010, 122 (Suppl 3):S768-786. 
8. Wolff B, Machill K, Schumacher D, Schulzki I, Werner D: Early achievement of mild therapeutic hypothermia and the neurologic outcome after cardiac arrest. Int J Cardiol 2009, 133:223-228

9. Haugk M, Testori C, Sterz F, Uranitsch M, Holzer M, Behringer W, Herkner H: Time to Target Temperature Study Group. Relationship between time to target temperature and outcome in patients treated with therapeutic hypothermia after cardiac arrest. Crit Care 2011, 15:R101

10. Neumar RW, Otto CW, Link MS, Kronick SL, Shuster M, Callaway CW, Kudenchuk PJ, Ornato JP, McNally B, Silvers SM, Passman RS, White RD, Hess EP, Tang W, Davis D, Sinz E, Morrison L: Part 8: adult advanced cardiovascular life support: 2010 American Heart Association Guidelines for Cardiopulmonary Resuscitation and Emergency Cardiovascular Care. Circulation 2010, 122 (Suppl 3):S729-767. Review. Erratum in: Circulation 2011, 123:e236

11. Pell JP, Sirel JM, Marsden AK, Ford I, Walker NL, Cobbe SM: Presentation, management, and outcome of out of hospital cardiopulmonary arrest: comparison by underlying aetiology. Heart 2003, 89:839-842.

12. Böttiger BW, Arntz HR, Chamberlain DA, Bluhmki E, Belmans A, Danays T, Carli PA, Adgey JA, Bode C, Wenzel V; TROICA Trial Investigators; European Resuscitation Council Study Group: Thrombolysis during resuscitation for out-of-hospital cardiac arrest. N Engl J Med 2008, 359:2651-2662.

13. Dumas F, Cariou A, Manzo-Silberman S, Grimaldi D, Vivien B, Rosencher J, Empana JP, Carli P, Mira JP, Jouven X, Spaulding C: Immediate percutaneous coronary intervention is associated with better survival after out-ofhospital cardiac arrest: insights from the PROCAT (Parisian Region Out of hospital Cardiac ArresT) registry. Circ Cardiovasc Interv 2010, 3:200-207.

14. Marcusohn E, Roguin A, Sebbag A, Aronson D, Dragu R, Amikam S, Boulus M, Grenadier E, Kerner A, Nikolsky E, Markiewicz W, Hammerman H, Kapeliovich M: Primary percutaneous coronary intervention after out-of-hospital cardiac arrest: patients and outcomes. Is Med Assoc J 2007, 9:257-259.

15. Knafelj R, Radsel P, Ploj T, Noc M: Primary percutaneous coronary intervention and mild induced hypothermia in comatose survivors of ventricular fibrillation with ST-elevation acute myocardial infarction. Resuscitation 2007, 74:227-234.

16. Wolfrum S, Pierau C, Radke PW, Schunkert $H$, Kurowski V: Mild therapeutic hypothermia in patients after out-of-hospital cardiac arrest due to acute ST-segment elevation myocardial infarction undergoing immediate percutaneous coronary intervention. Crit Care Med 2008, 36:1780-1786.

17. Gräsner JT, Meybohm P, Lefering R, Wnent J, Bahr J, Messelken M, Jantzen T, Franz R, Scholz J, Schleppers A, Böttiger BW, Bein B, Fischer M; German Resuscitation Registry Study Group: ROSC after cardiac arrest - the RACA score to predict outcome after out-of-hospital cardiac arrest. Eur Heart 2011, 32:1649-1656

18. Sunde K, Pytte M, Jacobsen D, Mangschau A, Jensen LP, Smedsrud C, Draegni T, Steen PA: Implementation of a standardised treatment protocol for post resuscitation care after out-of-hospital cardiac arrest. Resuscitation 2007, 73:29-39

19. Rivers EP, Rady MY, Martin GB, Fenn NM, Smithline HA, Alexander ME, Nowak RM: Venous hyperoxia after cardiac arrest. Characterization of a defect in systemic oxygen utilization. Chest 1992, 102:1787-1793.

20. Kilgannon JH, Jones AE, Shapiro NI, Angelos MG, Milcarek B, Hunter K, Parrillo JE, Trzeciak S, Emergency Medicine Shock Research Network (EMShockNet) Investigators: Association between arterial hyperoxia following resuscitation from cardiac arrest and in-hospital mortality. JAMA 2010, 303:2165-2171.

21. Pilcher J, Weatherall M, Shirtcliffe P, Bellomo R, Young P, Beasley R: The effect of hyperoxia following cardiac arrest - a systematic review and metaanalysis of animal trials. Resuscitation 2012, 83:417-422.

22. Bellomo R, Bailey M, Eastwood GM, Nichol A, Pilcher D, Hart GK, Reade MC, Egi M, Cooper D); Study of Oxygen in Critical Care (SOCC) Group: Arterial hyperoxia and in-hospital mortality after resuscitation from cardiac arrest. Crit Care 2011, 15:R90

23. Kolar M, Krizmaric M, Klemen P, Grmec S: Partial pressure of end-tidal carbon dioxide successful predicts cardiopulmonary resuscitation in the field: a prospective observational study. Crit Care 2008, 12:R115.

24. Weil MH: Partial pressure of end-tidal carbon dioxide predicts successful cardiopulmonary resuscitation in the field. Crit Care 2008, 12:90

25. Levine RL, Wayne MA, Miller CC: End-tidal carbon dioxide and outcome of out-of-hospital cardiac arrest. N Engl J Med 1997, 337:301-306.

26. Eckstein M, Hatch L, Malleck J, McClung C, Henderson SO: End-tidal CO2 as a predictor of survival in out-of-hospital cardiac arrest. Prehosp Disaster Med
2011, 26:148-150.

27. Grmec S, Lah K, Tusek-Bunc K: Difference in end-tidal $\mathrm{CO} 2$ between asphyxia cardiac arrest and ventricular fibrillation/pulseless ventricular tachycardia cardiac arrest in the prehospital setting. Crit Care 2003, 7:R139-144.

28. Lah K, Krizmaric M, Grmec S: The dynamic pattern of end-tidal carbon dioxide during cardiopulmonaryresuscitation: difference between asphyxial cardiac arrest and ventricular fibrillation/pulseless ventricular tachycardia cardiac arrest. Crit Care 2011, 15:R13.

29. International Liasion Committee on Resuscitation: 2005 International Consensus on Cardiopulmonary Resuscitation and Emergency Cardiovascular Care Science with Treatment Recommendations. Resuscitation 2005, 67:181-314.

30. Mattox KL, Beall AC Jr:. Resuscitation of the moribund patient using portable cardiopulmonary bypass. Ann Thorac Surg 1976, 22:436-442

31. Hill JG, Bruhn PS, Cohen SE, Gallagher MW, Manart F, Moore CA, Seifert PE, Askari P, Banchieri C: Emergent applications of cardiopulmonary support: a multiinstitutional experience. Ann Thorac Surg 1992, 54:699-704.

32. Martin GB, Rivers EP, Paradis NA, Goetting MG, Morris DC, Nowak RM: Emergency department cardiopulmonary bypass in the treatment of human cardiac arrest. Chest 1998, 113:743-751

33. Chen YS, Lin JW, Yu HY, Ko WJ, Jerng JS, Chang WT, Chen WJ, Huang SC, Chi $\mathrm{NH}$, Wang CH, Chen LC, Tsai PR, Wang SS, Hwang JJ, Lin FY: Cardiopulmonary resuscitation with assisted extracorporeal life-support versus conventional cardiopulmonary resuscitation in adults with in-hospital cardiac arrest: an observational study and propensity analysis. Lancet 2008, 372:554-561.

34. Kagawa E, Inoue I, Kawagoe T, Ishihara M, Shimatani Y, Kurisu S, Nakama Y, Dai K, Takayuki O, Ikenaga H, Morimoto Y, Ejiri K, Oda N: Assessment of outcomes and differences between in- and out-of-hospital cardiac arrest patients treated with cardiopulmonary resuscitation using extracorporeal life support. Resuscitation 2010, 81:968-973.

35. Shinar Z, Bellezzo J, Paradis N, Dembitsky W, Jaski B, Mallon W, Watt T: Emergency department initiation of cardiopulmonary bypass: a case report and review of the literature. J Emerg Med 2012, 43:83-86.

36. Arlt M, Philipp A, Voelkel S, Graf BM, Schmid C, Hilker M: Out-of-hospital extracorporeal life support for cardiac arrest-A case report. Resuscitation 2011, 82:1243-1245.

37. Le Guen M, Nicolas-Robin A, Carreira S, Raux M, Leprince P, Riou B, Langeron $\mathrm{O}$ : Extracorporeal life support following out-of-hospital refractory cardiac arrest. Crit Care 2011, 15:R29.

38. Valenzuela TD, Roe DJ, Cretin S, Spaite DW, Larsen MP: Estimating effectiveness of cardiac arrest interventions: a logistic regression survival model. Circulation 1997, 96:3308-3313.

39. Rea TD, Cook AJ, Stiell IG, Powell J, Bigham B, Callaway CW, Chugh S, Aufderheide TP, Morrison L, Terndrup TE, Beaudoin T, Wittwer L, Davis D, Idris A, Nichol G; Resuscitation Outcomes Consortium Investigators: Predicting survival after out-of-hospital cardiac arrest: role of the Utstein data elements. Ann Emerg Med 2010, 55:249-257.

40. Hayashi Y, Hiraide A, Morita H, Shinya H, Nishiuchi T, Yukioka H, Ikeuchi H, Matsusaka M, Shigemoto T, Ueshima T, Iwami T, Kai T, Fujii C: Three year longitudinal study for out-of-hospital cardiac arrest in Osaka Prefecture. Resuscitation 2004, 63:161-166.

41. Koike S, Ogawa T, Tanabe S, Matsumoto S, Akahane M, Yasunaga H, Horiguch $H$, Imamura T: Collapse-to-emergency medical service cardiopulmonary resuscitation interval and outcomes of out-of-hospital cardiopulmonary arrest: a nationwide observational study. Crit Care 2011, 15:R120.

42. Spaite DW, Hanlon T, Criss EA, Valenzuela TD, Wright AL, Keeley KT, Meislin HW: Prehospital cardiac arrest: the impact of witnessed collapse and bystander CPR in a metropolitan EMS system with short response times. Ann Emerg Med 1990, 19:1264-1269.

43. Sasson C, Rogers MA, Dahl J, Kellermann AL: Predictors of survival from out-of-hospital cardiac arrest: a systematic review and meta-analysis. Circ Cardiovasc Qual Outcomes 2010, 3:63-81.

44. Caffrey SI, Willoughby PJ, Pepe PE, Becker LB: Public use of automated defibrillators. N Engl J Med 2002, 347:1242-1247.

45. Weisfeldt ML, Sitlani CM, Ornato JP, Rea T, Aufderheide TP, Davis D, Dreyer J Hess EP, Jui J, Maloney J, Sopko G, Powell J, Nichol G, Morrison LJ; ROC Investigators: Survival after application of automatic external defibrillators before arrival of the emergency medical system: evaluation in the resuscitation outcomes consortium population of 21 million. J Am Coll 
Cardiol 2010, 55:1713-1720

46. Neukamm J, Graesner J, Schewe J, Breil M, Bahr J, Heister U, Wnent J, Bohn A Heller G, Strickmann B, Fischer H, Kill C, Messelken M, Bein B, Lukas R, Meybohm P, Scholz J, Fischer M: The impact of response time reliability on CPR incidence and resuscitation success - a benchmark study from the German Resuscitation Registry. Crit Care 2011, 15:R282.

47. Gräsner JT, Meybohm P, Fischer M, Bein B, Wnent J, Franz R, Zander J, Lemke H, Bahr J, Jantzen T, Messelken M, Dörges V, Böttiger BW, Scholz J: A national resuscitation registry of out-of-hospital cardiac arrest in Germany-a pilot study. Resuscitation 2009, 80:199-203.

48. Gräsner JT, Meybohm P, Caliebe A, Bottiger BW, Wnent J, Messelken M, Jantzen T, Zeng T, Strickmann B, Bohn A, Fischer H, Scholz J, Fischer M, German Resuscitation Registry Study Group: Postresuscitation care with mild therapeutic hypothermia and coronary intervention after out-ofhospital cardiopulmonary resuscitation: a prospective registry analysis. Crit Care 2011, 15:R61.

49. Nolan JP, Lockey D: Airway management for out-of-hospital cardiac arrest more data required. Resuscitation 2009, 80:1333-1334.

50. Kajino K, Iwami T, Kitamura T, Daya M, Ong ME, Nishiuchi T, Hayashi Y, Sakai T, Shimazu T, Hiraide A, Kishi M, Yamayoshi S: Comparison of supraglottic airway versus endotracheal intubation for the pre-hospital treatment of out-of-hospital cardiac arrest. Crit Care 2011, 15:R236.

51. Shy BD, Rea TD, Becker $L$, Eisenberg MS: Time to intubation and survival in prehospital cardiac arrest. Prehosp Emerg Care 2004, 8:394-399.
52. Dunham CM, Barraco RD, Clark DE, Daley BJ, Davis FE 3rd, Gibbs MA, Knuth T, Letarte PB, Luchette FA, Omert L, Weireter LJ, Wiles CE 3rd; EAST Practice Management Guidelines Work Group: Guidelines for emergency tracheal intubation immediately after traumatic injury. J Trauma 2003, 55:162-179.

53. Ruchholtz S, Waydhas C, Ose C, Lewan U, Nast-Kolb D; Working Group on Multiple Trauma of the German Trauma Society: Prehospital intubation in severe thoracic trauma without respiratory insufficiency: a matched-pair analysis based on the Trauma Registry of the German Trauma Society. J Trauma 2002, 52:879-886.

54. Hussmann B, Lefering R, Waydhas C, Ruchholtz S, Wafaisade A, Kauther MD Lendemans S: Prehospital intubation of the moderately injured patient: a cause of morbidity? A matched-pairs analysis of 1,200 patients from the DGU Trauma Registry. Crit Care 2011, 15:R207.

55. Mort TC: Complications of emergency tracheal intubation: hemodynamic alterations - part I. J Intensive Care Med 2007, 22:157-165.

56. Dutton RP, Mackenzie CF, Scalea TM: Hypotensive resuscitation during active hemorrhage: impact on in-hospital mortality. J Trauma 2002, 52:1141-1146.

doi:10.1186/cc11832

Cite this article as: Goldberg SA, et al.: Year in review 2011: Critical Care Out-of-hospital cardiac arrest and trauma. Critical Care 2012, 16:247. 